${ }^{1}$ E.-J. Freyse

${ }^{1} \mathrm{~K}$. Rebrin

${ }^{2}$ T. Schneider

${ }^{3}$ M. Petrzika

${ }^{1} \mathrm{U}$. Fischer

\section{Nitrogen economy in insulin-dependent, normoglycemic diabetic dogs}

Stickstoffökonomie bei diabetischen Hunden

\section{Problem}

Disturbances of protein metabolism (growth retardation) have been observed in young diabetics regularly treated with insulin. A close correlation between average blood glucose control and urinary nitrogen excretion has been previously reported, We could show that diabetic dogs maintained normoglycemic by means of portal (prehepatic) glucose-controlled insulin infusion exhibit normal gluconeogenic flux rates from alanine but that these rates were elevated with systemic (posthepatic) insulin administration. Therefore, the study was designed to investigate the influence of route of insulin administration (portal versus systemic) on the urea production rate, which is considered to indicate amino acid catabolism. In addition the impact of different food protein contents was elucidated.

\section{Materials and methods}

Paired studies were conducted in chronic insulin-dependent diabetic dogs. Diabetic dogs and nondiabetic controls were fed either a high-protein diet $(46 \%$ of energy intake provided by protein, study 1) or a low-protein carbohydrate-supplemented diet ( $20 \%$ of energy provided by pro-

\footnotetext{
${ }^{1}$ E.-J. Freyse - 1 K. Rebrin - ${ }^{2}$ T. Schneider - ${ }^{3}$ M. Petrzika

'U. Fischer

"Institut für Diabetes "Gerhardt Katsch"

17495 Karlsburg

Institut für Medizinische Genetik der Ernst-Moritz-Arndt-

Universität Greifswald

17495 Karlsburg

${ }^{3}$ Deutsches Institut für Ernährungsforschung

14558 Bergholz-Rehbrücke

Germany
}

tein, study 2) for two days. The flux rates of glucose and urea were measured using isotope dilution techniques. The diabetic dogs were maintained normoglycemic by glucose-controled insulin infusion delivered either systemically or portally.

\section{Results}

There were no significant differences in plasma glucose and glucose turnover between the experimental groups on either systemic or portal insulin infusion versus controls. Peripheral insulin levels were higher for diabetic animals maintained with systemic versus portal insulin delivery (p.05). In study 1 versus study 2 , the animals showed lower -amino nitrogen levels and concentrations of gluconeogenic amino acids, predominantly alanine (p.05). No significant differences in glucagon, lactate, pyruvate, nonesterified fatty acids, or B-hydroxybutyrate were observed. Urea production was significantly higher in study 1 compared with study $2: 7.48 \pm 0.83$ vs. $5.97 \pm 0$. $59 \mu \mathrm{mol} \mathrm{kg} \mathrm{kmin}^{-1}$ (normal dogs); $12.97 \pm 1.86$ vs. 5 . $54 \pm 0.60 \mu \mathrm{mol} \mathrm{kg}^{-1} \mathrm{~min}^{-1}$ (diabetic dogs on portal insulin) $16.11 \pm 2.59$ vs. $6.82 \pm 0.70 \mu \mathrm{mol}^{-\mathrm{kg}^{-1}} \mathrm{~min}^{-1}$ (diabetic dogs on systemic insulin infusion); p.05 for all. The diabetic dogs maintained normoglycemic with systemic insulin infusions had significantly higher rates of urea synthesis than those with portal insulin infusion $(\mathrm{p}<0.05)$.

\section{Conclusions}

The hepatic insulin to glucagon ratio caused by systemic insulin administration may be the primary event for increased urea production. According to the plasma amino acid data the difference in urea production is not caused by the availability of amino acids. In the future a more advanced metabolic management of insulin-dependent diabetes should be aimed to restore normal protein turnover. 